\title{
Efficient Dissolution of Partnerships and the Structure of Control
}

\author{
Emanuel Ornelas and John L. Turner*
}

January 29, 2004

\begin{abstract}
In this paper, we study efficient dissolution of partnerships in a context of incomplete information. We generalize the results of Cramton, Gibbons and Klemperer (1987) to situations where the partnership takes on a common value that may depend upon all partners' types, so that each partner's individual rationality constraint depends on types other than his own. We show that in this case not only the distribution of ownership, emphasized in the earlier literature, but also the distribution of control within an organization matter to determine the possibility of efficient dissolution. We underscore this point by showing that two-person partnerships where one partner exercises complete control cannot be dissolved efficiently with any incentive compatible, individually rational mechanism, regardless of the ownership structure.
\end{abstract}

JEL Code: C72, D82, L14

Keywords: Mechanism design, efficient trading, asymmetric control, partnerships.

\section{Introduction}

In 1999, Dave Aynes, 50\% owner of Blue Sky Coffee in Athens, GA, wished to buy out his partner Mark Fierer. According to their partnership agreement, dissolution was to occur by "Cowboy Shootout," where either partner can make an offer at any time but where any partner's offer for the other's shares is both a bid and ask. The responding partner can buy or sell at that price, but must choose one of these options. Expecting to buy, Dave shaded his bid for Mark's shares a bit below his own valuation for the business. Things degenerated quickly.

Mark was living in Los Angeles, CA, more than 3,000 miles away, at the time. Since Blue Sky was profitable, Mark's nominal role as a $50 \%$ partner ensured him a steady flow of income.

${ }^{*}$ Terry College of Business, University of Georgia, Fifth Floor, Brooks Hall, Athens, GA 30602-6254. Ornelas: 706-542-2284, eornelas@terry.uga.edu; Turner: 706-542-3682, jlturner@terry.uga.edu. We thank Sander Onderstal, Daniel Ferreira and Jacob Goeree for helpful comments 
Mark viewed Dave's offer as too low, and thus decided to buy out Dave at his offer price. Upon learning of the prospect of this potential buyout, some of Blue Sky's vendors indicated that they would be unwilling to maintain the same relationship with Blue Sky. ${ }^{1}$ This problem had not been foreseen in the partners' contractually specified dissolution mechanism. Ultimately, since it was in fact efficient for Dave, who lived in Athens and had emerged over time as a superior manager, to own Blue Sky, Dave renegotiated to buy out Mark, though at a higher price than his original offer. In the process, substantial wasted effort had been undertaken and feelings had been hurt.

At first glance, this case seems to illustrate the shortcomings of the Cowboy Shootout as a dissolution mechanism. ${ }^{2}$ Since Mark's value for the firm was above Dave's bid (but almost surely beneath Dave's valuation), his attempt to buy out Dave reflects the inherent inefficiency of this dissolution mechanism. However, as we argue in this paper, it is unlikely that any individually rational dissolution mechanism would have worked efficiently in view of the way their partnership was functioning. Specifically, provided that their valuations for Blue Sky under the partnership were interdependent - as it would be the case if they valued the firm according to its flows of profits - and since Dave was exercising virtually complete control over the management of the firm, it would be impossible to design an individually rational mechanism that ensured an efficient dissolution of the partnership.

Theoretical inquiries into the efficient dissolution of partnerships have shown that asymmetric information and asymmetric ownership shares both make it difficult and sometimes impossible to design individually rational mechanisms to implement efficient dissolution. Akerlof (1970) provided the fundamental intuition for the effects of asymmetric information in an extremeownership setting, and Myerson and Satterthwaite (1983) proved general impossibility results for bilateral exchange under private information. Subsequent work, particularly that of Cramton, Gibbons and Klemperer (1987, henceforth "CGK"), has shown that, among partners with independent and identically distributed signals, the Myerson-Satterthwaite impossibility result extends to partnerships where the partners' shares are unbalanced though not necessarily extreme. CGK point out, however, that equal-shares partnerships can always be dissolved efficiently while unequal-shares partnerships can be dissolved efficiently provided that ownership is not "too unbalanced."

In recent work, Jehiel and Pauzner (2002), Moldovanu (2002) and Fieseler, Kittsteiner and Moldovanu (2003) consider instead the case of interdependent private valuations. They all show that interdependent private valuations can affect the set of dissolvable partnerships significantly. Fieseler et al. and Moldovanu find that, when information is ex-ante symmetric, a partnership

\footnotetext{
${ }^{1}$ For instance, Blue Sky's landlord refused to negotiate a new lease in Mark's name.

${ }^{2}$ Moldovanu (2002) refers to this type of mechanism as the "Texas Shootout;" McAfee (1992) proves that it is generally inefficient.
} 
is more difficult to dissolve if a given partner's valuation is increasing in the types of the other partners, while the opposite is true if the partner's valuation is decreasing in the signals of the other partners. In the former case, the equal-shares partnership may not be dissolvable efficiently, while in the latter case efficient bilateral exchange may be possible. Jehiel and Pauzner focus on cases where only one partner is informed about the value of the co-owned asset. In such a setting, they identify a wide class of situations where efficient dissolution is unachievable.

In the models of all papers cited above, individual rationality requires that any partner aware of his private signal earns, via the dissolution mechanism, at least as much as his share times his implied expected valuation. This modeling choice neglects one potentially crucial feature of partnerships - that an entity's gross value, from an individual partner's perspective, may depend on the very existence of the partnership. That is, for any partner, the entity/asset may take on different gross values depending on whether it remains a partnership or it is dissolved.

Circumstances where this occurs are natural in many trading situations. Consider, for instance, the case of a regular business whose value stems from the flow of profits it generates. In such a case, the independent valuation of each partner would reflect the profits he could make with full ownership — and thus complete control — of the business's assets. ${ }^{3}$ Under a partnership, by contrast, control is generally distributed among the partners, giving rise to a different administration and thus a distinct flow of profits. Such profits represent the value of the assets under the partnership and are common to all partners. Individual rationality then requires that any partner aware of his private signal earns, via the dissolution mechanism, at least as much as his share times his expected valuation for the business were it to remain a partnership. This view contrasts with the conventional notion in the literature, where valuations do not depend on the distribution of control. ${ }^{4}$

Considering that partners are restricted to using individually rational, incentive compatible mechanisms, in the tradition of the literature, we provide general conditions governing when efficient dissolution of partnerships that share the features described above are possible. Specifically, each partner has an independent type that represents his private valuation for the asset if it is owned individually. Each partner draws his type from distributions that share a common support, but we allow them to be otherwise different. Under the partnership, the asset provides a common value for all partners, which may depend on their independent private valuations.

\footnotetext{
${ }^{3}$ Control represents in this paper what Aghion and Tirole (1997) have described as real authority. We employ a distinct terminology to distinguish our paper from the literature spurred by Aghion and Tirole's seminal paper, since we focus on distinct issues from those emphasized in that line of research - e.g., we do not attempt to explain how real authority is acquired or when it is likely to be detached from formal authority.

${ }^{4}$ A related but distinct approach is taken by Jehiel and Pauzner (2002), who allow for "increasing returns to scale" in the ownership shares. That is, they allow each partner's valuation for the asset to increase disproportionately with the partner's share of the asset. In such a case, valuations depend on the ownership structure. We posit that valuations may depend also on the firm's structure of control, which may or may not be related with the ownership structure.
} 
Thus, our structure can be viewed as a hybrid of the independent private values case studied by CGK and the interdependent valuations case examined by Jehiel and Pauzner (2002), Moldovanu (2002) and Fieseler et al. (2003). The distinctive feature of our setting is that here "sole proprietor" valuations are independent, while valuations under co-ownership are common.

In general, the common value of a partnership depends on how its assets are managed. Since several forms of management are possible, co-owners must decide which to employ. Such a decision may depend on a myriad of features related to the partnership-background and experience of the partners, the distribution of shares among them, as well as the issues shaping real authority mentioned by Aghion and Tirole (1997). Explaining this choice is, however, beyond the scope of this paper, where we take the distribution of control, and the resulting interdependent individual valuations for the partnership, as given.

While we provide general conditions for dissolvability of partnerships that share the characteristics just described, we highlight our main point by focusing the balance of our analysis on a particular form of arrangement, which is similar to that of Blue Sky. Specifically, one partner runs the business independently, while the other partners share the profits but do not have any influence on its management. We term this type of control structure (in the absence of a better name) a "silent partnership," where one partner is "active" and all others are "silent" (in the case of Blue Sky, Dave served as the "active" partner and Mark as the single "silent" partner). In such a case, the gross value of the entity as a partnership, common to all partners, is simply the active partner's valuation, since his valuation corresponds to what he would obtain by owning, and thus controlling, the entity solely.

We show that there is not any incentive compatible, individually rational mechanism that can dissolve efficiently silent partnerships that consist of only two partners. While surprising in view of previous results in the literature, this finding highlights how the distribution of control in organizations can affect the prospects of dissolution. The asymmetry of control, emphasized at the extreme in a silent partnership, thus emerges as another potential stumbling block for efficient dissolution of partnerships.

We also find, however, that as the number of partners increases, the problems stemming from asymmetry of control may be mitigated, making efficient dissolution "easier" (in a sense to be made clear in Section 3) to achieve. In fact, with more than two partners even extreme ownership partnerships can be dissolvable, as long as the effective owner of the business - the one who owns all of its assets - is a silent partner; if the effective owner is instead the active partner, the Myerson-Satterthwaite impossibility result obtains.

We also analyze how the relative "abilities" of partners affect dissolvability. We show that, provided that the ownership share of the active partner is not too large, having a "better" active partner makes dissolution more difficult, while having "better" silent partners makes dissolution easier. We conclude with an example that illustrates each of our key results. 
The balance of this paper is structured as follows. In Section 2, we derive conditions governing efficient dissolution of partnerships when valuations are common under co-ownership but independent otherwise. In Section 3, we apply our results to study the structure of silent partnerships. Section 4 concludes.

\section{The Model}

The conventional notion of individual rationality is that any partner aware of his valuation of the entity earns, via the dissolution mechanism, at least as much as his share (say $r_{i}$ ) times his valuation (say $v_{i}$ ), namely $U_{i}\left(v_{i}\right) \geq r_{i} v_{i}$. This notion of individual rationality is appropriate in some situations but not in others, ${ }^{5}$ as for example when the partnership is a business whose value derives from the flow of profits it generates for the partners. In situations like that, the value of the assets for all partners under the partnership are likely to be interdependent - and possibly common. At a general level, we can define this value as a common function of all partners' individual valuations, namely $v_{P}=v_{P}\left(v_{1}, \ldots, v_{n}\right)$. Thus, to participate in a dissolution mechanism, each partner will require at least his share $r_{i}$ of the firm's value $v_{P}$-which in general is different from $v_{i}$. That is, the individual rationality constraint that needs to be satisfied for each partner is not $U_{i}\left(v_{i}\right) \geq r_{i} v_{i}$, but $U_{i}\left(v_{i}\right) \geq r_{i} v_{P}$.

The actual form of $v_{P}$ will ultimately depend on how the organization is managed by the partners and how they interact in exercising control. We could have, in particular, either $v_{P}>\max \left\{v_{i}, \ldots, v_{n}\right\}$ or $v_{P} \leq \max \left\{v_{i}, \ldots, v_{n}\right\}$. The former case corresponds to situations where interaction among the partners creates a positive externality (i.e., there is "synergy"), while the latter case may represent situations where the partners do not work well together, or where a partner who is not the most capable "manager" among them detains full control. We develop our general results for efficient dissolution of partnerships without restricting the form of $v_{P}$. We nevertheless note that it will be efficient to dissolve a partnership only when $v_{P} \leq \max \left\{v_{i}, \ldots, v_{n}\right\}$, so the following analysis is relevant only to those circumstances. When that condition is met, a dissolution mechanism will be efficient if it allocates the entity to the partner with the highest valuation with probability one.

\footnotetext{
${ }^{5}$ For instance, suppose two individuals share the purchase price of a recreational motorboat as well as the fees required to dock it in a marina that they both use. They furthermore divide the rights to use the boat according to their shares. If they later decide to dissolve the partnership by having one partner sell out, the conventional notion of individual rationality is sensible. In maintaining the partnership as it is, each partner derives a particular enjoyment $v_{i}$ from the boat, and receives that enjoyment at any given moment with probability $r_{i}$. Therefore, for each partner's participation in the dissolution mechanism to be individually rational, inequality $U_{i}\left(v_{i}\right) \geq r_{i} v_{i}$ must hold.
} 


\subsection{Preliminaries}

A firm is jointly owned by $n$ risk-neutral partners indexed by $i \in\{1, \ldots, n\}$. Partner $i$ owns a share $r_{i} \in[0,1]$ of the partnership; shares sum to $1\left(\sum_{i=1}^{n} r_{i}=1\right)$. Partner $i$ has type $v_{i}$, where $v_{i} \in[\underline{v}, \bar{v}]$. Each $v_{i}$ is drawn independently from distribution $F_{i}$, which is common knowledge and has positive continuous density $f_{i}$. Each type $v_{i}$ represents the firm's flow of profits under partner $i$ 's sole administration. Intuitively, it can be understood as a measure of partner $i$ 's "managerial capacity."

Under the partnership, the firm generates aggregate profits that define a common gross value for the partners, $v_{P}=v_{P}\left(v_{1}, \ldots, v_{n}\right)$. This value will depend on the (exogenously given) distribution of control and on the (quality of the) interaction among the partners effectively controlling the firm. Profits are distributed to the partners according to their shares in the firm. For example, partner $i$ receives $v_{P} r_{i}$.

Using the revelation principle, we focus on a direct revelation game where partners report simultaneously their valuations $v=\left\{v_{1}, \ldots, v_{n}\right\}$ and a mechanism allocates shares $s(v)=$ $\left\{s_{1}, \ldots, s_{n}\right\}$ and determines transfer payments $t(v)=\left\{t_{1}, \ldots, t_{n}\right\}$ to the players. We restrict attention to mechanisms that are budget balanced-i.e., which satisfy $\sum s_{i}(v)=1$ and $\sum t_{i}(v)=0$. We refer to $\langle s, t\rangle$ as a trading mechanism.

Under the mechanism, partner $i$ obtains utility $v_{i} s_{i}+t_{i}$. Let $-i \equiv N \backslash i$ and let $E_{-i}\{\cdot\}$ denote the expectation operator with respect to $v_{-i}$. A generic partner $i$ expects to receive shares and transfers $S_{i}\left(v_{i}\right) \equiv E_{-i}\left\{s_{i}\left(v_{i}\right)\right\}$ and $T_{i}\left(v_{i}\right) \equiv E_{-i}\left\{t_{i}\left(v_{i}\right)\right\}$, respectively. His expected utility from the mechanism is therefore given by $U_{i}\left(v_{i}\right)=v_{i} S_{i}\left(v_{i}\right)+T_{i}\left(v_{i}\right)$. By contrast, partner $i$ 's expected utility under the partnership is $r_{i} P_{i}(v)$, where $P_{i}(v) \equiv E_{-i}\left\{v_{P}(v)\right\}$. In the remainder of this section, we provide the conditions for efficient dissolution of such partnerships. Since our distinct set of individual rationality constraints does not require altering the CGK methodology significantly, we follow it closely.

\subsection{Conditions for Efficient Dissolution of a Partnership}

We restrict attention to incentive compatible mechanisms. Incentive compatibility requires

$$
U_{i}\left(v_{i}\right) \geq v_{i} S_{i}(u)+T_{i}(u) \text { for all } i, v_{i}, u \in[\underline{v}, \bar{v}] .
$$

In turn, a trading mechanism $\langle s, t\rangle$ is individually rational if each of the partners expects to receive a non-negative net payoff from participating in the mechanism:

$$
U_{i}\left(v_{i}\right) \geq r_{i} P_{i}(v) \text { for all } i, v_{i} \in[\underline{v}, \bar{v}] .
$$

This is the condition that distinguishes this paper from previous studies. Essentially, partners' 
valuations are interdependent if the partnership is kept but independent if the partnership is dissolved. This perspective contrasts with the viewpoint of Myerson and Satterthwaite (1983) and CGK, who consider valuations to be always independent, as well as with that of Fieseler et al. (2003), Jehiel and Pauzner (2002) and Moldovanu (2002), who consider valuations to be always interdependent.

In such a setting, the following lemmas specify necessary and sufficient conditions for a mechanism to be incentive compatible and individually rational. First, since the conditions required for incentive compatibility do not depend upon the individual rationality constraint, they are the same as in CGK.

Lemma 1 A trading mechanism $\langle s, t\rangle$ is incentive compatible if and only if, for every $i \in N$, $S_{i}$ is increasing and

$$
T_{i}\left(v_{i}^{*}\right)-T_{i}\left(v_{i}\right)=\int_{v_{i}^{*}}^{v_{i}} u d S_{i}(u)
$$

for all $v_{i}, v_{i}^{*} \in[\underline{v}, \bar{v}]$.

Proof. See CGK, pp. 626-27.

Henceforth, we require the following condition to be satisfied:

Condition $1 P_{i}^{\prime}(v) \equiv d P_{i}(v) / d v_{i} \geq 0$ and $P_{i}^{\prime \prime}(v) \equiv d^{2} P_{i}(v) / d v_{i}^{2}<S_{i}^{\prime}\left(v_{i}\right) \forall i$.

Condition 1 asserts that the expected value of the partnership is weakly increasing in each partner's type. Intuitively, this simply indicates that profits under the partnership tend to be (weakly) higher when the owners are "more capable." The condition also asserts that $P_{i}($.$) must$ be either concave or not "too convex" in each $v_{i}$ (since Lemma 1 requires $S_{i}^{\prime} \geq 0$ ), so the function displays either decreasing or not-too-increasing marginal returns. ${ }^{6}$

Condition 1 is necessary for our characterization of the worst-off type of trader for each partner. Characterizing the worst-off type is important because it defines a lower bound for the individual rationality constraint: if it pays for the worst-off type to participate in the mechanism, it pays for all other types as well.

Lemma 2 Given an incentive compatible mechanism $\langle s, t\rangle$ such that the expected share function is monotone increasing and continuous on $[\underline{v}, \bar{v}]$, trader $i^{\prime} s$ net utility achieves a minimum at $v_{i}^{*}$, where $v_{i}^{*}$ is implicitly defined by

$$
S_{i}\left(v_{i}^{*}\right)=r_{i} P_{i}^{\prime}\left(v_{i}^{*}, v_{-i}\right) .
$$

\footnotetext{
${ }^{6}$ Note that, under CGK's standard notion of individual rationality, $P_{i}(v)=v_{i}$, so Condition 1 is trivially satisfied.
} 
Proof. The minimization of $U_{i}\left(v_{i}\right)-r_{i} P_{i}(v)$ is characterized by first-order necessary condition $U_{i}^{\prime}\left(v_{i}\right)=r_{i} P_{i}^{\prime}(v)$. But from Lemma 1, it follows that $U_{i}^{\prime}\left(v_{i}\right)=S_{i}\left(v_{i}\right)$. Hence, the net expected gain from the mechanism is minimized at $v_{i}^{*}$ such that $S_{i}\left(v_{i}^{*}\right)=r_{i} P_{i}^{\prime}\left(v_{i}^{*}, v_{-i}\right)$, since the secondorder condition for a minimum, $S_{i}^{\prime}\left(v_{i}\right)>r_{i} P^{\prime \prime}\left(v_{i}^{*}\right)$, is satisfied by Condition 1 and because $S_{i}^{\prime}\left(v_{i}\right) \geq 0$ from Lemma 1 .

In the present setting, the worst-off type may expect to be either a seller or a buyer under the mechanism. Such an expectation depends on how $P_{i}^{\prime}\left(v_{i}^{*}, v_{-i}\right)$ compares with unity. If $P_{i}^{\prime}\left(v_{i}^{*}, v_{-i}\right)=1$, as in CGK, the worst-type expects to be neither a seller nor a buyer. By contrast, it expects to be a buyer (seller) whenever $P_{i}^{\prime}\left(v_{i}^{*}, v_{-i}\right)>1(<1)$.

We can now establish necessary and sufficient conditions for individual rationality.

Lemma 3 An incentive compatible mechanism $\langle s, t\rangle$ is individually rational if and only if, for all $i \in N$,

$$
T_{i}\left(v_{i}^{*}\right) \geq r_{i}\left[P_{i}\left(v_{i}^{*}, v_{-i}\right)-v_{i}^{*} P_{i}^{\prime}\left(v_{i}^{*}, v_{-i}\right)\right] .
$$

Proof. We need only check individual rationality for the worst-off types $\left\{v_{i}^{*}\right\}$. The constraint for the worst-type is

$$
v_{i}^{*} S_{i}\left(v_{i}^{*}\right)+T_{i}\left(v_{i}^{*}\right) \geq r_{i} P_{i}\left(v_{i}^{*}, v_{-i}\right) .
$$

Using Lemma 2, it is straightforward to see that this condition is equivalent to equation 5 .

We are now ready to characterize the set of dissolvable partnerships.

Lemma 4 For any share function $s$ such that $S_{i}$ is increasing for all $i \in N$, there exists a transfer function $t$ such that $\langle s, t\rangle$ is incentive compatible and individually rational if and only if

$$
\sum_{i=1}^{n}\left[\int_{v_{i}^{*}}^{\bar{v}}\left[1-F_{i}(u)\right] u d S_{i}(u)-\int_{\underline{v}}^{v_{i}^{*}} F_{i}(u) u d S_{i}(u)\right] \geq \sum_{i=1}^{n} r_{i}\left[P_{i}\left(v_{i}^{*}, v_{-i}\right)-v_{i}^{*} P_{i}^{\prime}\left(v_{i}^{*}, v_{-i}\right)\right] .
$$

Proof. See appendix.

A mechanism $\langle s, t\rangle$ is ex-post efficient if the partnership is sold to the partner with the highest private valuation. Thus, we have the following modified version of CGK's Theorem 1:

Theorem 1 A partnership with ownership rights $\left\{r_{i}\right\}$ and types $\left\{v_{i}\right\}$ independently drawn from $F_{i}$ can be dissolved efficiently if and only if

$$
\sum_{i=1}^{n}\left[\int_{v_{i}^{*}}^{\bar{v}}\left[1-F_{i}(u)\right] u d G_{i}(u)-\int_{\underline{v}}^{v_{i}^{*}} F_{i}(u) u d G_{i}(u)\right] \geq \sum_{i=1}^{n}\left[r_{i} P_{i}\left(v_{i}^{*}, v_{-i}\right)-v_{i}^{*} G_{i}\left(v_{i}^{*}\right)\right],
$$

where $v_{i}^{*} \in \arg \min _{v_{i} \in[\underline{v}, \bar{v}]}\left[U_{i}\left(v_{i}\right)-r_{i} P_{i}(v)\right]$ and $G_{i}\left(v_{i}\right) \equiv \prod_{j \neq i} F_{j}\left(v_{i}\right)$. 
Proof. See appendix.

Theorem 1 characterizes the set of dissolvable partnerships. Within our framework, the results of CGK form an important special case in which the right-hand side of equation 7 is nil and distributions $F_{i}$ are identical for all $i$. We next introduce the notion of silent partnerships and study the circumstances when their efficient dissolution can be accomplished.

\section{Asymmetric Control and The Silent Partnership}

There are many possible situations in which one partner exercises disproportionate control of the partnership's operations. The simplest and perhaps most common occurs when, in order to finance a venture, an entrepreneur sells equity in his venture to investors who have little interest in managing the partnership. ${ }^{7}$ To analyze the impact of asymmetric control on dissolvability, we focus on an important benchmark case, which we term the "silent partnership."

\subsection{Definition and Basic Rationale}

In a silent partnership, one "active" partner has full control over the business, managing it on behalf of himself and the other "silent" partners. Each partner has an independent private signal that represents what the value for the firm would be if he were to be its sole proprietor. Because of the silent partnership structure, however, the value of the firm as a partnership is given by the active partner's signal, since he alone controls the firm's operation.

Definition 1 (Silent Partnership - $S P\left\langle n, r, F_{1}, F_{2}\right\rangle$ ) Let partner 1 have full control over the jointly owned business; call him the "active" partner and all other $n-1$ partners the "silent" partners. Let $r$ denote the active partner's share of the partnership; thus, the silent partners' shares sum to $(1-r)$. Let the active partner's signal $v_{1}$ be drawn from distribution $F_{1}$ and the silent partners signals $\left\{v_{2}, \ldots, v_{n}\right\}$ be each drawn from distribution $F_{2}$. All $v_{i}$ are drawn independently. Furthermore, distributions $F_{1}$ and $F_{2}$ have a mutual support $[\underline{v}, \bar{v}]$. The value of the firm under the partnership is defined as $v_{P}\left(v_{1}, \ldots, v_{n}\right)=v_{1}$.

Under this structure, whenever the signal of any silent partner is higher than the active partner's signal, an efficient dissolution of the partnership is Pareto improving. Generally, if the active partner's private valuation is high (low), he will wish to buy out (sell to) the silent partners. A silent partner with a high valuation will also wish to buy out his partners. However, if his valuation is low, he does not wish to sell; rather, he prefers to keep the partnership intact.

\footnotetext{
${ }^{7}$ This was, in fact, the original reason why Mark Fierer became a partner in Blue Sky.
} 
Thus, given the partners' potential contrasting incentives to seek dissolution, it is important to strive for efficient mechanisms to accomplish this.

Note that, under our structure, the active partner's signal may have a different distribution than the silent partners' signals. As such, our main impossibility results are robust to situations where the active partner is believed to be a "better" or "worse" manager than the silent partners as well as to situations where the partners have had the opportunity to learn about the active partner's skills. Moreover, this allows us to analyze whether more capable partners make dissolution easier.

In an $S P, P_{1}\left(v_{1}^{*}, v_{-1}\right)=v_{1}^{*}$ and $P_{i}\left(v_{i}^{*}, v_{-i}\right)=\int_{\underline{v}}^{\bar{v}} u d F_{1}(u)$ for all $i \neq 1$. Moreover, $v_{1}^{*}=G_{1}^{-1}(r)$ and $v_{i}^{*}=\underline{v}$ for all $i \neq 1$. Using this information, we can apply Theorem 1 to find that a silent partnership can be efficiently dissolved when:

$$
\begin{array}{r}
\left\{\int_{G_{1}^{-1}(r)}^{\bar{v}}\left[1-F_{1}(u)\right] u d G_{1}(u)-\int_{\underline{v}}^{G_{1}^{-1}(r)} F_{1}(u) u d G_{1}(u)\right\}+(n-1) \int_{\underline{v}}^{\bar{v}}\left[1-F_{2}(u)\right] u d G_{2} \\
\geq(1-r) \int_{\underline{v}}^{\bar{v}} u d F_{1}(u)
\end{array}
$$

where $G_{1}(u)=F_{2}(u)^{n-1}$ and $G_{2}(u)=F_{1}(u) F_{2}(u)^{n-2}$. The terms on the left-hand side of the inequality give the sum of the expected transfers to the worst-off types of partners. Not including possible side payments, the term in braces is the expected transfer $\left(T_{1}^{*}\right)$ to the worst-off type of active partner $\left(G_{1}^{-1}(r)\right)$. Note that this type of active partner will be, on average, neither a buyer nor a seller under the mechanism. This property holds for all worst-off types in the setting analyzed by CGK. Intuitively, this type is the worst-off because he would reveal his type truthfully without any incentives and thus receives no informational rent.

The worst-off type of silent partner $(\underline{v})$, on the other hand, is independent of the distribution of shares. As such, the sum of the expected transfers to the worst-off types of silent partners, not including side payments, is just $(n-1)$ times the expected transfer to any one of them. Note that the worst-off type of any silent partner expects to sell his shares with certainty.

The term on the right-hand side of the inequality is the sum of the expected profits that would accrue to the silent partners were the partnership to remain intact. Note that only the size, and not the distribution, of the $(1-r)$ share of the partnership among the silent partners matters for dissolvability. Since the worst-off type of silent partner sells his shares with certainty, this term equals exactly the minimum total compensation that the silent partners need to receive under the mechanism to be willing to participate.

In proving our results, we make frequent use of the following Lemma, which presents a simplified version of condition 8. 
Lemma 5 An $S P\left\langle n, r, F_{1}, F_{2}\right\rangle$ can be dissolved efficiently if and only if

$$
\int_{G_{1}^{-1}(r)}^{\bar{v}}\left[r-G_{1}(u)\right] d u+\int_{\underline{v}}^{\bar{v}}\left\{1-r-(n-1) F_{2}(u)^{n-2}\left[1-F_{2}(u)\right]\right\} F_{1}(u) d u \geq 0
$$

Proof. See Appendix.

\subsection{Impossibility Results}

Under the partnership structure $S P\left\langle n, r, F_{1}, F_{2}\right\rangle$, the inherent asymmetry of control affects crucially the possibility of constructing efficient dissolution mechanisms. In particular, when there are only two partners, efficient dissolution is impossible regardless of the distribution of ownership shares. In addition, as in Myerson and Satterthwaite (1983) and CGK, extreme asymmetric ownership makes efficient dissolvability/exchange more difficult to achieve. In our setting, when the active partner owns the entire firm $(r=1)$, efficiency cannot be achieved. However, efficiency becomes possible when a silent partner owns the entire firm. We present each set of results in turn.

Proposition 1 An $S P\left\langle n=2, r, F_{1}, F_{2}\right\rangle$ cannot be dissolved efficiently with an incentive compatible, individually rational mechanism.

Proof. We will show that inequality 9 does not hold in this case. Note that in a $S P$ with $n=2$, $G_{1}(u)=F_{2}(u)$. Thus, when $n=2$, we can rewrite condition 9 as

$$
\int_{F_{2}^{-1}(r)}^{\bar{v}}\left[r-F_{2}(u)\right] d u+\int_{\underline{v}}^{\bar{v}}\left[F_{2}(u)-r\right] F_{1}(u) d u \geq 0 .
$$

This inequality can be re-arranged as

$$
\int_{F_{2}^{-1}(r)}^{\bar{v}}\left[r-F_{2}(u)\right]\left[1-F_{1}(u)\right] d u+\int_{\underline{v}}^{F_{2}^{-1}(r)}\left[F_{2}(u)-r\right] F_{1}(u) d u \geq 0 .
$$

It is easy to see that both terms in the left-hand side of this inequality are non-positive and at least one must be strictly negative. Specifically, since $F_{2}(u)>r$ when $u>F_{2}^{-1}(r)$,

$$
\int_{F_{2}^{-1}(r)}^{\bar{v}}\left[r-F_{2}(u)\right]\left[1-F_{1}(u)\right] d u \leq 0
$$

where the inequality is strict if $r<1$. Similarly, since $F_{2}(u)<r$ when $u<F_{2}^{-1}(r)$,

$$
\int_{\underline{v}}^{F_{2}^{-1}(r)} F_{1}(u)\left[F_{2}(u)-r\right] d u \leq 0
$$


where the inequality is strict if $r>0$. Therefore, for $r \in[0,1]$, neither term in the left-hand side of inequality 10 is positive and at least one is strictly negative. It follows that Theorem 1 does not hold for an $S P\left\langle n=2, r, F_{1}, F_{2}\right\rangle$.

Intuitively, dissolution is impossible here precisely because the worst-off type of silent partner expects to sell to the active partner with certainty. He will only wish to participate if he expects to be paid a price (per share) that is at least as large as the active partner's (expected) valuation. But since it is impossible to get truthful revelation from the active partner without giving him some informational rent, a positive outside subsidy is necessary to make participation individually rational for all types.

Such a subsidy is also necessary to achieve efficient "dissolution" when the active partner owns the entire firm.

Proposition 2 An $S P\left\langle n, r=1, F_{1}, F_{2}\right\rangle$ cannot be dissolved efficiently with an incentive compatible, individually rational mechanism.

Proof. When $r=1$, condition 9 reduces to

$$
-(n-1) \int_{\underline{v}}^{\bar{v}} F_{1}(u) F_{2}(u)^{n-2}\left[1-F_{2}(u)\right] d u \geq 0,
$$

which is a contradiction, thus completing the proof.

Notice that expression 11 is, for $n=2$, identical to Myerson and Satterthwaite's (1983) equation 7 . In our setting, this also represents the minimum outside subsidy necessary to facilitate efficient dissolution. Indeed, dissolution is impossible in this particular case for precisely the same reasons as in Myerson and Satterthwaite (1983). Intuitively, any incentive compatible mechanism requires a transfer to be made regardless of whether the active partner sells the firm. Thus, when the active partner is the worst-off type $(\bar{v})$, he knows that, under the mechanism, he must make a payment despite the fact that he will maintain full ownership with certainty. The expected gains to the worst-off types of silent partners are not large enough to produce a big enough side payment from the silent partners to the active partner to induce the worst-off active partner to participate.

In contrast to Myerson and Satterthwaite (1983) and CGK, however, when the partnership is owned entirely by one silent partner $(r=0)$, efficient "dissolution" may be possible. This is seen as a simple consequence of our finding that, for $r<1$, dissolvability is always possible if $n$ is large enough.

Proposition 3 Any $S P\left\langle n, r<1, F_{1}, F_{2}\right\rangle$ can be dissolved efficiently with an incentive compatible, individually rational mechanism for sufficiently large $n$. 
Proof. We will show that condition 9 can always be satisfied when $n$ becomes arbitrarily large and $r<1$. In that case, $G_{1}^{-1}(r) \equiv\left(F_{2}^{n-1}\right)^{-1}(r)$ becomes arbitrarily close to $\bar{v}$, $G_{1}(r) \equiv F_{2}^{n-1}(u)$ becomes arbitrarily close to zero and the first integral in 9 vanishes. Furthermore, since $\lim _{n \rightarrow \infty}(n-1) F_{2}(u)^{n-2}\left[1-F_{2}(u)\right] F_{1}(u)=0$, the second integral in 9 specializes to $\int_{\underline{v}}^{\bar{v}}(1-r) F_{1}(u) d u$. Thus, when $n \rightarrow \infty$ (and $r<1$ ), condition 9 simplifies to

$$
(1-r) \int_{\underline{v}}^{\bar{v}} F_{1}(u) d u \geq 0
$$

which is satisfied for any distribution $F_{1}(u)$.

If $r=0$, the worst-off type $(\underline{v})$ is the same for all partners, and each of these types expects to sell their shares with certainty. When $n>2$, there are at least two silent partners. Thus, if there is a full-ownership silent partner and he happens to be the worst-off type, he expects to sell to the active partner with some probability and expects to sell to one of the other silent partners with some probability. Thus, in any efficient mechanism, he sells at a price that exceeds, in expected terms, the active partner's valuation. Hence, his expected payoff may, after factoring in informational rents, exceed his payoff from his continued ownership. The worst-off type of active partner, knowing that with certainty he will not buy the firm, needs no incentives to participate in the mechanism.

It is worth noting that when $n$ increases, the additional "partners" need not own positive shares in the firm. In fact, they may simply represent additional bidders for the partnership. Interestingly, then, for $r<1$ it may be possible to overcome the impossibility result for $n=2$ if the two owners are willing to permit outsiders to buy the firm.

Note, however, that while any $S P$ with $r<1$ can be dissolved for large enough $n$, it is not always the case that dissolvability becomes easier with a larger $n$. In particular, when $r$ is near 1 and $n$ is small, it is straightforward to find distributions of the partners' signals such that dissolvability becomes more difficult with an additional partner. We explore this issue further with an example in subsection 3.4 .

\subsection{Comparative Statics}

When $n>2$ and $r<1$, whether a silent partnership is dissolvable depends upon the number of partners $n$, the active partner's share $r$ and the distribution of the partners' signals $F_{1}$ and $F_{2}$. In this and the next subsection, we show how changes in each of these primitives affect dissolvability. When characterizing the set of dissolvable partnerships, we will refer frequently to the following definition.

Definition 2 Given two silent partnerships $S P$ and $S P^{*}$, we say that $S P^{*}$ is "easier" to dissolve 
than $S P$ if and only if the left-hand side of inequality 9 (in Lemma 5) is larger for $S P^{*}$.

Thus, if $S P$ is dissolvable and $S P^{*}$ is easier to dissolve, then $S P^{*}$ must also be dissolvable. By the same token, if $S P^{*}$ is easier to dissolve than $S P$ but $S P^{*}$ is not dissolvable without a positive outside subsidy, then $S P$ requires a larger outside subsidy to be dissolvable.

Given this definition, in the setting of CGK the equal-shares partnership is the easiest to dissolve. This is not typically true in a silent partnership, although it is still true that the extreme-ownership settings $(r=0$ and $r=1)$ are never the easiest to dissolve.

Proposition 4 Let $\mu_{1} \equiv \int_{\underline{v}}^{\bar{v}} u d F_{1}(u)$. If partnership $S P\left\langle n, r, F_{1}, F_{2}\right\rangle$ can be dissolved efficiently for some $r$, then partnership $S P\left\langle n, r=G_{1}\left(\mu_{1}\right), F_{1}, F_{2}\right\rangle$ can be dissolved efficiently too. Similarly, if partnership $S P\left\langle n, r=G_{1}\left(\mu_{1}\right), F_{1}, F_{2}\right\rangle$ cannot be dissolved efficiently, then partnership $S P\left\langle n, r, F_{1}, F_{2}\right\rangle$ cannot be dissolved efficiently for any $r$.

Proof. It is sufficient to show that the left-hand side of condition 9 is maximized when $r=$ $G_{1}\left(\mu_{1}\right)$. Differentiating that expression with respect to $r$, we find

$$
\begin{aligned}
\frac{d L H S(9)}{d r} & =\int_{G_{1}^{-1}(r)}^{\bar{v}} d u+\frac{d G_{1}^{-1}(r)}{d r}\left[r-G_{1}\left(G_{1}^{-1}(r)\right)\right]-\int_{\underline{v}}^{\bar{v}} F_{1}(u) d u \\
& =\bar{v}-G_{1}^{-1}(r)-\left(\bar{v}-\mu_{1}\right) \\
& =\mu_{1}-G_{1}^{-1}(r) .
\end{aligned}
$$

Since this expression is decreasing in $r\left(d G_{1}^{-1}(r) / d r>0\right)$, the left-hand side of condition 9 is maximized when $\mu_{1}=G_{1}^{-1}(r)$ - or equivalently, when $r=G_{1}\left(\mu_{1}\right)$, completing the proof.

Hence, for given $n, F_{1}$ and $F_{2}$, the partnership with $r=G_{1}\left(\mu_{1}\right)$ is the easiest to dissolve. Ownership shares affect dissolution in two different ways in this setting. On the one hand, a greater $r$ decreases the expected transfer to the worst-off type of active partner at a rate $G_{1}^{-1}(r)$, just as it would do under CGK's setting. The worst-off type of silent partners are, however, qualitatively distinct. Their expected transfers do not increase with $r$ [i.e. as their $(1-r)$ share decreases], as they would under CGK's setting. Rather, their participation costs fall with $r$, at a constant rate $\mu_{1}$. Conditions for efficient dissolution are facilitated at the extreme when these two rates are equalized, as proved above. ${ }^{8}$

In a sense, this insight resembles a key result from CGK, namely that efficient dissolution tends to become easier to achieve as the ownership structure becomes less extreme. Indeed, it is clear that, for any finite $n$ and non-degenerate $F_{2}$ distribution, $0<G_{1}\left(\mu_{1}\right)<1$, so the most

\footnotetext{
${ }^{8}$ In CGK, the two correspondent rates (for the 2-player case) would be $G^{-1}(r)$ and $G^{-1}(1-r)$-recall that they consider identical distributions. Thus, dissolution is facilitated at the extreme when $G^{-1}(r)=G^{-1}(1-r)$, which occurs when $r=\frac{1}{2}$.
} 
extreme-ownership settings are indeed never the easiest to dissolve. However, it is also clear that the equal-shares partnership will not generally be the easiest to dissolve, as $G_{1}\left(\mu_{1}\right)$ depends on $F_{1}, F_{2}$ and $n$.

Since the impossibility of dissolving two-person silent partnerships is due, essentially, to possible free-riding by the silent partner, it is to be expected that, as the benefits from free-riding increases, dissolution would become more difficult. Intuitively, free-riding by silent partners becomes a more attractive option for them as the distribution of the active partner's signal becomes "better" or as the distribution of the silent partner's signal becomes "worse." We now analyze these effects in turn.

First, we show that the presence of an active partner who is a "better" manager makes dissolution more difficult whenever $r$ is not too large.

Proposition 5 Consider distributions $F_{1}^{*}$ and $F_{1}$ such that $F_{1}^{*}$ first-order stochastically dominates $F_{1}$ and define $\widetilde{r}(n) \equiv 1-\left(\frac{n-2}{n-1}\right)^{n-2}$. If partnership $S P\left\langle n>2, r \leq \widetilde{r}(n), F_{1}^{*}, F_{2}\right\rangle$ can be dissolved efficiently, then partnership $S P\left\langle n>2, r \leq \widetilde{r}(n), F_{1}, F_{2}\right\rangle$ can be dissolved efficiently too. Similarly, if partnership $S P\left\langle n>2, r \leq \widetilde{r}(n), F_{1}, F_{2}\right\rangle$ cannot be dissolved efficiently, then partnership $S P\left\langle n>2, r \leq \widetilde{r}(n), F_{1}^{*}, F_{2}\right\rangle$ cannot be dissolved efficiently either.

Proof. See Appendix.

When $v_{1}$ is drawn from $F_{1}^{*}$ instead of $F_{1}$, where $F_{1}^{*}$ first-order stochastically dominates $F_{1}$, there is no change from the perspective of an active partner of a given type. By contrast, for the silent partners, incentive compatibility becomes less costly while individual rationality becomes more costly to achieve. The former effect occurs because the informational rents required to induce truth-telling by the silent partners fall when $v_{1}$ is drawn from $F_{1}^{*}$ instead of $F_{1} .{ }^{9}$ The latter effect occurs because the participation costs are higher under $F_{1}^{*}$ than under $F_{1}$. Intuitively, when the active partner draws from a first-order stochastically dominating distribution, his expected signal is higher, and so is the value he engenders into the firm. As a result, the worstoff type of silent partner becomes more content to free ride off the efforts of the active partner, making his participation in the dissolution mechanism more costly.

The change in the informational rents is independent of $r$, but the change in the participation costs is inversely related to $r$. Thus, when $r$ approaches 1 , the reduction in informational rents predominates over the increase in participation costs, making the partnership easier to dissolve. By contrast, for moderate values of $r$ (i.e., when $r<\widetilde{r}$ ), the increase in participation costs dominates and the partnership becomes more difficult to dissolve when $v_{1}$ is drawn from $F_{1}^{*}$ instead of $F_{1}$.

\footnotetext{
${ }^{9}$ To see this, note that a silent partner with type $v_{s}$ obtains informational rents equal to $\int_{\underline{v}}^{v_{s}} F_{1}(u) F_{2}(u)^{n-2} d u$, which is clearly lower under $F_{1}^{*}$ than under $F_{1}$.
} 
Note also that, as the active partner's expected signal increases, the gains from dissolving the partnership fall, since it becomes less likely that a silent partner will be more efficient than the active partner in controlling the business. This rationale highlights a more general feature of silent partnerships: they tend to be more difficult to dissolve precisely when dissolution is less desirable, from an efficiency perspective.

It is interesting to note that the increase in participation costs always predominates in the benchmark case of equal-shares partnerships, as the corollary below shows.

Corollary 1 Consider distributions $F_{1}^{*}$ and $F_{1}$ such that $F_{1}^{*}$ first-order stochastically dominates $F_{1}$. If an equal-shares partnership $S P\left\langle n>2, r=\frac{1}{n}, F_{1}^{*}, F_{2}\right\rangle$ can be dissolved efficiently, then partnership $S P\left\langle n>2, r=\frac{1}{n}, F_{1}, F_{2}\right\rangle$ can be dissolved efficiently too. Similarly, if partnership $S P\left\langle n>2, r=\frac{1}{n}, F_{1}^{*}, F_{2}\right\rangle$ cannot be dissolved efficiently, then partnership $S P\left\langle n>2, r=\frac{1}{n}, F_{1}, F_{2}\right\rangle$ cannot be dissolved efficiently either.

Proof. See Appendix.

We now analyze the intuitive counterpart to Proposition 5-i.e., the conditions where a "worse" silent partner makes dissolvability more difficult. We show that this will happen whenever $r$ is not too large.

Proposition 6 Consider distributions $F_{2}^{*}$ and $F_{2}$ such that $F_{2}^{*}$ first-order stochastically dominates $F_{2}$ and define $\bar{r}$ implicitly by

$$
\int_{G_{1}^{-1}(\bar{r})}^{\bar{v}} F_{2}(u)^{n-2} d u=-\int_{\underline{v}}^{\bar{v}} F_{1}(u) F_{2}(u)^{n-2}\left[(n-2) F_{2}(u)^{-1}\left(1-F_{2}(u)\right)-1\right] d u .
$$

$\bar{r}$ is greater than zero and may be greater than one. If partnership $S P\left\langle n>2, r \leq \bar{r}, F_{1}, F_{2}\right\rangle$ can be dissolved efficiently, then partnership $S P\left\langle n>2, r \leq \bar{r}, F_{1}, F_{2}^{*}\right\rangle$ can be dissolved efficiently too. Similarly, if partnership $S P\left\langle n>2, r \leq \bar{r}, F_{1}, F_{2}^{*}\right\rangle$ cannot be dissolved efficiently, then partnership $S P\left\langle n>2, r \leq \bar{r}, F_{1}, F_{2}\right\rangle$ cannot be dissolved efficiently either.

Proof. See Appendix.

Proposition 6 shows that "worse" silent partners tend to make it more difficult to dissolve a partnership whenever the share owned by the active partner is not too high $(r \leq \bar{r})$. When the silent partners' signals are drawn from $F_{2}$ instead of $F_{2}^{*}$, where $F_{2}^{*}$ first-order stochastically dominates $F_{2}$, there is no change in the individually rationality constraints. By contrast, the incentive compatibility constraints of all partners are affected. For the silent partners, incentive compatibility becomes more costly to achieve, since the informational rents required to induce truth-telling by the silent partners increase when their signals are drawn from $F_{2}$ instead of $F_{2}^{*}$ 
(this follows straightforwardly from footnote 9). This change is independent of $r$. For the active partner, on the other hand, the change in incentive compatibility will generally depend on $r{ }^{10}$

When $r=0$, the effect is unambiguous: informational rents increase for all partners, and dissolution becomes more difficult when the silent partners' signals are drawn from a first-order stochastically dominated distribution function. The same applies when $r$ is sufficiently small $(r \leq \bar{r})$, but not necessarily otherwise.

\subsection{Examples}

To further illuminate these results, we consider the class of examples where distributions display the following forms:

$$
\begin{aligned}
& F_{1}\left(v_{1}\right)=v_{1}^{\alpha} \text { for } v_{1} \in[0,1], \alpha>0 \\
& F_{2}\left(v_{i}\right)=v_{i}^{\beta} \text { for } v_{i} \in[0,1], \beta>0 .
\end{aligned}
$$

In this case, condition 8 becomes:

$$
\begin{aligned}
(n-1)\{\beta & {\left[\frac{1}{\beta(n-1)+1}-\frac{1}{\alpha+\beta(n-1)+1}-\frac{r^{\frac{\beta(n-1)+1}{\beta(n-1)}}}{\beta(n-1)+1}\right] } \\
& \left.+(\beta(n-2)+\alpha)\left[\frac{1}{\alpha+\beta(n-2)+1}-\frac{1}{\alpha+\beta(n-1)+1}\right]\right\} \geq(1-r) \frac{\alpha}{1+\alpha}
\end{aligned}
$$

Note that when $\alpha=\beta=1$, all partners' valuations are iid uniform on [0,1]. Condition 14 then reduces to

$$
(n-1)\left(\frac{1}{1+n}-\frac{r^{\frac{n}{n-1}}}{n}\right) \geq \frac{1-r}{2} .
$$

The curved surface in Figure 1 plots the left-hand side minus the right-hand side of the above expression, for $r \in[0,1]$ and $n=\{2,3, \ldots, 100\}$. The flat plane is at zero for all $r$ and $n$. Hence, when the surface is above the plane, the inequality is satisfied.

When $n=2$, inequality 15 is not satisfied for any distribution of shares. When $n=3$, all partnerships with $r \leq \frac{9}{16}$ are dissolvable, while all partnerships with $r>\frac{9}{16}$ are not. Note that, in contrast to CGK, the range of dissolvable partnerships is not symmetric about the equalshares partnership. As such, there are ownership structures where partnerships are dissolvable here but not dissolvable in the setting of CGK and vice-versa. Most notably, the inequality above is always satisfied when $n>2$ and $r=0$, so this "extreme ownership" partnership is

\footnotetext{
${ }^{10}$ To see this, note that an active partner with type $v_{1}$ obtains informational rents equal to $\int_{G_{1}^{-1}(r)}^{v_{1}}\left[G_{1}(u)-\right.$ $r] d u+r v_{1}$. It can be easily shown that, when we replace $F_{2}^{*}$ by $F_{2}$, the change in this expression decreases with $r$ if and only if $v_{1} \geq v_{1}^{*}$.
} 


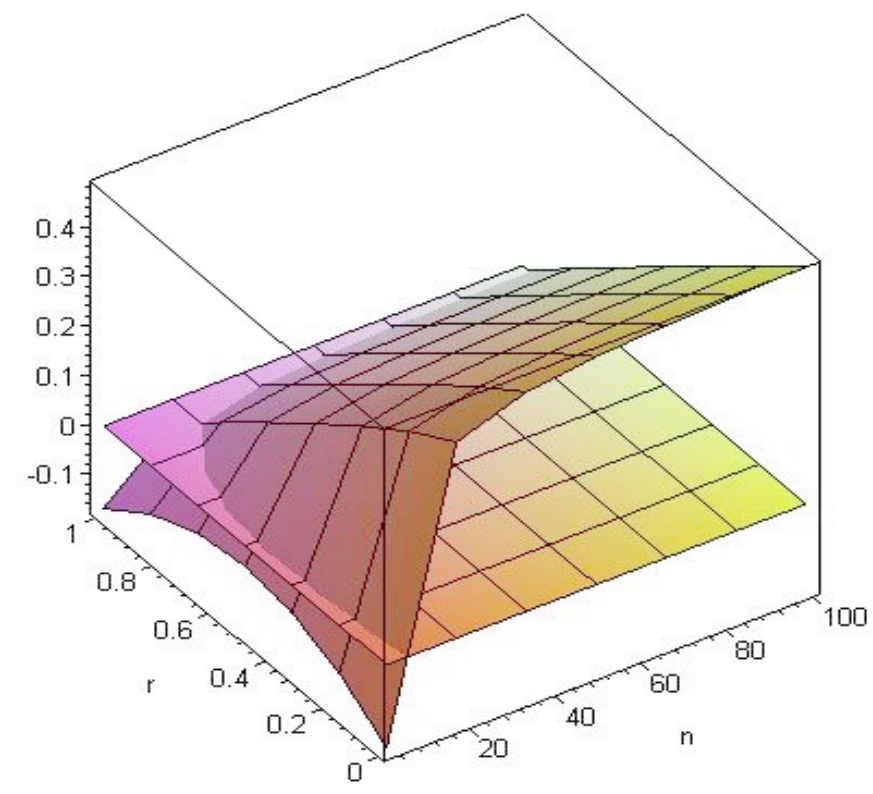

Figure 1: Efficient Dissolution with Uniform Distributions

dissolvable unless $n=2 .{ }^{11}$ On the other hand, even though the upper bound of the range of dissolvable partnerships increases with $n$, it is not satisfied for any $n$ when $r=1$.

Now, to illustrate Proposition 5 and Corollary 1 , we fix $\beta=1$ and analyze changes in $\alpha$. Note that when $\alpha>(<) 1$, the active partner is expected to have a higher (lower) signal than any silent partner. Focusing on the case of equal-shares partnerships $\left(r=\frac{1}{n}\right)$, the inequality above is satisfied, when $n=3$ if and only if $\alpha \leq 1.8$ (approximately). In this case, the higher is $\alpha$ (the more capable is the active partner), the more difficult the partnership is to dissolve.

To illustrate Proposition 6 , we fix $\alpha=1$ and analyze changes in $\beta$. Note that when $\beta>(<) 1$, all silent partners are expected to have a higher (lower) signal than the active partner. For the equal-shares partnerships $\left(r=\frac{1}{n}\right)$, the inequality above is satisfied, when $n=3$, if and only if $\beta \geq .64$ (approximately). In this case, the lower is $\beta$ (the less capable are the silent partners), the more difficult the partnership is to dissolve.

In this class of examples, for most values of $r$, partnerships become easier to dissolve with additional silent partners. This is true for both of the cases just discussed. When $r=\frac{1}{3}, \alpha=1.8$ and $\beta=1$, partnerships with $n=4$ are easier to dissolve than partnerships with $n=3$. The same holds if $\alpha=1$ and $\beta=.64$. However, for large $r$, this is not necessarily true. For the latter

\footnotetext{
${ }^{11}$ By contrast, the partnership with ownership shares $\left\{r_{1}=\frac{5}{8}, r_{2}=\frac{3}{8}, r_{3}=0\right\}$ is dissolvable under CGK but not dissolvable here, since $\frac{5}{8}>\frac{9}{16}$.
} 
case, if $r=.95$, a subsidy of approximately .124 is necessary to dissolve the partnership if $n=3$, but .125 is needed if $n=4$.

\section{Conclusion}

We have demonstrated another potential obstacle to efficient dissolution of partnerships, namely asymmetry of control of a firm's operations. Our results suggest that partnerships in which one partner dominates the active management of the firm will often encounter problems when they attempt to dissolve. This problem is most acute if there is only one "silent" partner. On the other hand, firms can mitigate this problem if they are willing, during the dissolution process, to entertain bidding by outsiders. The intuition from this case is likely to extend to less extreme control structures.

Numerous papers have addressed the determinants of real authority/control structure within organizations. Other papers have studied the forces shaping the efficient dissolution of partnerships. However, none of the contributions in each of these lines of research has analyzed the effects of the structure of control on the design of efficient dissolution mechanisms. This paper starts to fill this gap. We provide a general framework for analysis and explore in detail a form of partnership that is characterized by an extreme but common form of control structure. Our general framework can nevertheless be applied to numerous other partnership structures. Further applications will likely help us access in more detail how the allocation of control within organizations may affect the prospects of efficient dissolution. We look forward to further progress in this area.

\section{Appendix}

Proof of Lemma 4. Only if. CGK (pp. 627-28) shows that incentive compatibility (Lemma 1) implies

$$
\sum_{i=1}^{n} T_{i}\left(v_{i}^{*}\right)=\sum_{i=1}^{n}\left[\int_{v_{i}^{*}}^{\bar{v}}\left[1-F_{i}(u)\right] u d S_{i}(u)-\int_{\underline{v}}^{v_{i}^{*}} F_{i}(u) u d S_{i}(u)\right] .
$$

Individual rationality (Lemma 3 ) implies, in turn, that

$$
\sum_{i=1}^{n} T_{i}\left(v_{i}^{*}\right) \geq \sum_{i=1}^{n} r_{i}\left[P_{i}\left(v_{i}^{*}, v_{-i}\right)-v_{i}^{*} P_{i}^{\prime}\left(v_{i}^{*}, v_{-i}\right)\right] .
$$

Hence, any $\langle s, t\rangle$ that is incentive compatible and individually rational must satisfy equation 6 . 
If. Following CGK (p. 628), consider a transfer of the form

$$
t_{i}(v)=c_{i}-\int_{\underline{v}}^{v_{i}} u d S_{i}(u)+\frac{1}{n-1} \sum_{j \neq i} \int_{\underline{v}}^{v_{j}} u d S_{j}(u),
$$

where $\sum_{i=1}^{n} t_{i}(v)=0$ implies $\sum_{i=1}^{n} c_{i}=0$. After changing the order of integration, we obtain

$$
T_{i}\left(v_{i}\right)=c_{i}-\int_{\underline{v}}^{v_{i}} u d S_{i}(u)+\frac{1}{n-1} \sum_{j \neq i} \int_{\underline{v}}^{v_{j}}\left[1-F_{i}(u)\right] d S_{j}(u) .
$$

This guarantees that the mechanism is incentive compatible.

Individual rationality requires

$$
T_{i}\left(v_{i}^{*}\right) \geq r_{i}\left[P_{i}\left(v_{i}^{*}, v_{-i}\right)-v_{i}^{*} P_{i}^{\prime}\left(v_{i}^{*}, v_{-i}\right)\right] .
$$

Since equation 6 asserts that

$$
\sum_{i=1}^{n} T_{i}\left(v_{i}^{*}\right) \geq \sum_{i=1}^{n} r_{i}\left[P_{i}\left(v_{i}^{*}, v_{-i}\right)-v_{i}^{*} P_{i}^{\prime}\left(v_{i}^{*}, v_{-i}\right)\right],
$$

we can choose

$$
\begin{aligned}
c_{i}=r_{i}\left[P_{i}\left(v_{i}^{*}, v_{-i}\right)-v_{i}^{*} P_{i}^{\prime}\left(v_{i}^{*}, v_{-i}\right)\right]+ & \frac{1}{n} \sum_{i=1}^{n}\left\{T_{i}\left(v_{i}^{*}\right)-r_{i}\left[P_{i}\left(v_{i}^{*}, v_{-i}\right)-v_{i}^{*} P_{i}^{\prime}\left(v_{i}^{*}, v_{-i}\right)\right]\right\} \\
& +\int_{\underline{v}}^{v_{i}^{*}} u d S_{i}(u) d u-\frac{1}{n-1} \sum_{j \neq i} \int_{\underline{v}}^{\bar{v}}\left[1-F_{i}(u)\right] u d S_{j}(u),
\end{aligned}
$$

which results in

$$
\begin{array}{r}
T_{i}\left(v_{i}^{*}\right)=r_{i}\left[P_{i}\left(v_{i}^{*}, v_{-i}\right)-v_{i}^{*} P_{i}^{\prime}\left(v_{i}^{*}, v_{-i}\right)\right]+\frac{1}{n} \sum_{i=1}^{n}\left\{T_{i}\left(v_{i}^{*}\right)-\right. \\
\left.r_{i}\left[P_{i}\left(v_{i}^{*}, v_{-i}\right)-v_{i}^{*} P_{i}^{\prime}\left(v_{i}^{*}, v_{-i}\right)\right]\right\} \\
\geq r_{i}\left[P_{i}\left(v_{i}^{*}, v_{-i}\right)-v_{i}^{*} P_{i}^{\prime}\left(v_{i}^{*}, v_{-i}\right)\right],
\end{array}
$$

completing the proof.

Proof of Theorem 1. Efficiency requires the partner with the highest independent private valuation to buy the firm. The probability that an individual partner's $v_{i}$ is the highest is $F_{i}\left(v_{i}\right)^{n-1}$. This imposes the following restriction on the share function: $S_{i}\left(v_{i}\right)=G_{i}\left(v_{i}\right)=$ $F_{i}\left(v_{i}\right)^{n-1}$. The worst-off type $v_{i}^{*}$ is defined according to Lemma 2 . 
Proof of Lemma 5. Rewrite condition 9 as

$$
\int_{G_{1}^{-1}(r)}^{\bar{v}} u d G_{1}(u)+\int_{\underline{v}}^{\bar{v}} u\left\{(n-1)\left[1-F_{2}(u)\right] d G_{2}(u)-F_{1}(u) d G_{1}(u)-(1-r) d F_{1}(u)\right\} \geq 0 .
$$

Integrating by parts each of the integrals above, the inequality can be rearranged as

$$
\begin{aligned}
{\left[\bar{v}-G_{1}^{-1}(r) r-\int_{G_{1}^{-1}(r)}^{\bar{v}} G_{1}(u) d u\right] } & \\
+ & {\left[\int_{\underline{v}}^{\bar{v}}\left\{1-r-(n-1) F_{2}(u)^{n-2}\left[1-F_{2}(u)\right]\right\} F_{1}(u) d u-(1-r) \bar{v}\right] \geq 0, }
\end{aligned}
$$

or equivalently as

$$
\int_{G_{1}^{-1}(r)}^{\bar{v}}\left[r-G_{1}(u)\right] d u+\int_{\underline{v}}^{\bar{v}}\left\{1-r-(n-1) F_{2}(u)^{n-2}\left[1-F_{2}(u)\right]\right\} F_{1}(u) d u \geq 0,
$$

thus proving the lemma.

Proof of Proposition 5. Since distribution $F_{1}^{*}$ first-order stochastically dominates distribution $F_{1}, F_{1}^{*}(u) \leq F_{1}(u)$ for all $u$. Note now that, in condition 9 , only the second integral depends on $F_{1}$, where $F_{1}$ enters multiplying the term in braces. The term in braces, in turn, is positive if and only if

$$
r \leq 1-(n-1) F_{2}(u)^{n-2}\left[1-F_{2}(u)\right] \equiv h\left(F_{2}(u)\right) .
$$

Function $h\left(F_{2}(u)\right)$ achieves a minimum at

$$
F_{2}(u)=\frac{n-2}{n-1}
$$

when it equals

$$
h\left(\frac{n-2}{n-1}\right)=1-\left(\frac{n-2}{n-1}\right)^{n-2} \equiv \widetilde{r}(n) .
$$

Hence, the second integral in condition 9 is always positive when $r \leq \widetilde{r}(n)$ and its value increases with $F_{1}(u)$. This implies that, when $r \leq \widetilde{r}(n)$, the left-hand side of condition 9 is greater under $F_{1}$ than under $F_{1}^{*}$. It follows that, if $S P\left\langle n, r<\widetilde{r}(n), F_{1}^{*}, F_{2}\right\rangle$ is dissolvable, $S P\left\langle n, r<\widetilde{r}(n), F_{1}, F_{2}\right\rangle$ is dissolvable too. Similarly, if $S P\left\langle n, r<\widetilde{r}(n), F_{1}, F_{2}\right\rangle$ is not dissolvable, $S P\left\langle n, r<\widetilde{r}(n), F_{1}^{*}, F_{2}\right\rangle$ is not dissolvable either.

Proof of Corollary 1. We need to show only that equal-shares partnerships fit the condition required in Proposition 5 about $r$. This is the case if $\frac{1}{n} \leq \widetilde{r}(n) \equiv 1-\left(\frac{n-2}{n-1}\right)^{n-2}$ when $n>2$. 
Rewriting this inequality as $\left(\frac{n-2}{n-1}\right)^{n-2} \frac{n}{n-1} \leq 1$, it is easy to see that its left-hand side decreases with $n$. Since the inequality is satisfied when $n=3\left(\frac{3}{4} \leq 1\right)$, the proof is complete.

Proof of Proposition 6. Since distribution $F_{2}^{*}$ first-order stochastically dominates distribution $F_{2}, F_{2}^{*}(u) \leq F_{2}(u)$ for all $u$. Note now that, as $F_{2}(u)$ increases at the margin for all $u$, the change in the left-hand side of condition 9 (LHS-9) corresponds to

$$
\begin{aligned}
& \frac{d \text { LHS-9 }}{d F_{2}(u)}= \\
-(n-1) & \int_{G_{1}^{-1}(r)}^{\bar{v}} F_{2}(u)^{n-2} d u-(n-1) \int_{\underline{v}}^{\bar{v}} F_{1}(u) F_{2}(u)^{n-2}\left[(n-2) F_{2}(u)^{-1}\left(1-F_{2}(u)\right)-1\right] d u,
\end{aligned}
$$

which is negative iff

$$
\int_{G_{1}^{-1}(r)}^{\bar{v}} F_{2}(u)^{n-2} d u \geq-\int_{\underline{v}}^{\bar{v}} F_{1}(u) F_{2}(u)^{n-2}\left[(n-2) F_{2}(u)^{-1}\left(1-F_{2}(u)\right)-1\right] d u \leq 0 .
$$

When $r=0$, this inequality becomes simply

$$
\int_{\underline{v}}^{\bar{v}} F_{2}(u)^{n-2}\left[(n-2) F_{2}(u)^{-1}\left(1-F_{2}(u)\right) F_{1}(u)+1-F_{1}(u)\right] d u \geq 0,
$$

so it clearly holds in that case. More generally, it is negative whenever $r \leq \bar{r}$. To see this, it suffices to note that $G_{1}^{-1}(\bar{r})$ increases with $r$, so it follows from definition 13 that $r \leq \bar{r}$ implies inequality 9 .

Thus, by continuity, LHS-9 $\left(r \leq \bar{r}, F_{2}^{*}\right) \geq \operatorname{LHS}-9\left(r \leq \bar{r}, F_{2}\right)$. It follows that LHS-9 $(r \leq$ $\left.\bar{r}, F_{2}^{*}\right) \leq 0$ implies LHS-9 $\left(r \leq \bar{r}, F_{2}\right) \leq 0$, so if partnership $S P\left\langle n>2, r \leq \bar{r}, F_{1}, F_{2}^{*}\right\rangle$ cannot be dissolved efficiently, partnership $S P\left\langle n>2, r \leq \bar{r}, F_{1}, F_{2}\right\rangle$ cannot be dissolved efficiently either. Similarly, LHS-9 $\left(r \leq \bar{r}, F_{2}\right) \geq 0$ implies LHS-9 $\left(r \leq \bar{r}, F_{2}^{*}\right) \geq 0$, so if partnership $S P\left\langle n>2, r \leq \bar{r}, F_{1}, F_{2}\right\rangle$ can be dissolved efficiently, partnership $S P\left\langle n>2, r \leq \bar{r}, F_{1}, F_{2}^{*}\right\rangle$ can be dissolved efficiently too.

\section{References}

Aghion, Philippe and Jean Tirole. "Formal and Real Authority in Organizations," Journal of Political Economy 105, 1997, pp. 1-29.

Akerlof, George. "The Market for Lemons: Quality Uncertainty and the Market Mechanism," Quarterly Journal of Economics 89, 1970, pp. 488-500. 
Cramton, Peter, Robert Gibbons and Paul Klemperer. "Dissolving a Partnership Efficiently," Econometrica 55 (3), 1987, pp. 615-632.

Fieseler, Karsten, Thomas Kittsteiner and Benny Moldovanu. "Partnerships, Lemons and Efficient Trade," Journal of Economic Theory 113, 2003, pp. 223-34.

Jehiel, Philippe and Ady Pauzner. "Partnership Dissolution with Interdependent Values," mimeo, 2002.

McAfee, Preston. "Amicable Divorce: Dissolving a Partnership with Simple Mechanisms," Journal of Economic Theory 56 (2), 1992, pp. 266-93.

Moldovanu, Benny. "How to Dissolve a Partnership," Journal of Institutional and Theoretical Economics 158, 2002, pp. 66-80.

Myerson, Roger B. and Mark Satterthwaite. "Efficient Mechanisms for Bilateral Trading," Journal of Economic Theory 29, 1983, pp. 265-81. 